\section{Hereditary disorders of surfactant homeostasis cause acute and chronic lung disease in infancy}

\author{
Jeffrey A Whitsett
}

In this issue of Thorax, Doan et al report the clinical and pathological findings from nine paediatric patients with severe acute and chronic lung disease caused by mutations in the ATP-binding cassette A3 protein (ABCA3), a lamellar body-associated transport protein expressed selectively in type II epithelial cells in the alveoli (see page 366). Previous reports regarding the mutations in $\mathrm{ABCA} 3$ were derived primarily from the analysis of term infants presenting with severe respiratory failure in the neonatal period. ${ }^{23}$ In the study by Doan et al, five of nine infants had respiratory symptoms at birth but only one died during the neonatal period. The others presented with chronic respiratory signs and symptoms at 3 months to 4 years of age, indicating the marked variability in age and severity of presentation of this genetic disease. These infants generally fail to thrive. Clinical, radiographic and pulmonary function testing were consistent with diffuse alveolar disease. Hypoxaemia and pulmonary function studies indicated severe restrictive lung disease. Lung pathology was commonly termed endogenous lipid pneumonia, pulmonary alveolar proteinosis, desquamative interstitial pneumonitis and nonspecific interstitial pneumonitis. The variability seen in the clinical presentation and pathological findings are perhaps related to age and treatments, as well as to the mutations inherited by each of the infants. Only one of the infants has died from the disorder. Three have received lung transplants and the others have had severe but relatively stable interstitial lung disease, clearly distinguishing this group of patients with ABCA3 generelated lung disease from newborn infants

Correspondence to: $\operatorname{Dr} J$ A Whitsett, Section of Neonatology, Perinatal and Pulmonary Biology, Cincinnati Children's Hospital Medical Center, 3333 Burnet Avenue, MLC 7029, Cincinnati, OH 45229-3039, USA; jeff. whitsett@cchmc.org presenting with fatal respiratory distress following birth.

ABCA3-related lung disease, like those caused by mutations in surfactant protein B (SFTPB) and surfactant protein C (SFTPC), demonstrate the critical importance of alveolar type II cell homeostasis in the pathogenesis of alveolar and interstitial lung disease..$^{2-7}$ Each of these genes is expressed selectively in type II alveolar epithelial cells, the site of surfactant synthesis. Proteins encoded by each gene regulate distinct aspects of the surfactant system, and each mediates distinct intracellular and extracellular activities related to surfactant function and/or lipid homeostasis. Together, these genetic defects in the surfactant system provide strong support for the concept that disruption of alveolar epithelial cell function causes chronic cell injury or cell death resulting in disturbed surfactant protein and lipid homeostasis, thus providing a paradigm for understanding the pathogenesis of acute and chronic lung diseases that have previously been considered idiopathic. It will be of interest to identify new genes within the pathways regulating alveolar type II cell function and surfactant homeostasis as a cause of other genetic disorders. Likewise, these genes represent potential proteins whose functions and expression may be altered secondarily by other inflammatory processes leading to acute and chronic lung disease.

ABCA3 is expressed selectively-but not specifically-in type II epithelial cells in the lung where it is detected at high levels in limiting membranes of lamellar bodies. Marked disruption of the morphology of lamellar bodies was observed by ultrastructural studies of tissue from patients with ABCA3-related lung disease. Likewise, decreased surfactant lipid content and abnormal intracellular and extracellular structure of lung lipids were associated with the disorder, as described herein and previously, and all consistent with the proposed role of ABCA3 in lipid transport. ${ }^{1} 2$ 8-10 Numerous distinct mutations in ABCA3 (a large and complex gene/protein) have been identified and associated with severe lung disease. Since no common mutations have been identified to date, the genetic diagnosis of ABCA3 is relatively difficult to make with certainty unless known mutations are identified. The present report provides strong clinical, radiographic and pathological correlates of ABCA3 deficiency that should be helpful in considering the diagnosis of this disorder. A definitive diagnosis will be helpful in the clinical management, genetic counselling and prenatal diagnosis of ABCA3-related disease. The diversity of age and clinical presentation of the lung disease associated with mutations in the ABCA3 gene suggest that environmental factors and other inherited genes may strongly influence the disease.

The study of surfactant homeostasis has provided ongoing insights into the important role of surfactant proteins and lipids that are required for lung function after birth. ABCA3 joins a number of other genes-including solute carrier 34 , a phosphate transporter recently associated with pulmonary microlithiasis, ${ }^{11}$ SFTPB and SFTPC $^{6}$-as genes encoding proteins critical for type II alveolar cell function related to surfactant homeostasis. While these disorders are generally rare genetic causes of alveolar and interstitial lung disease, together they strongly support the critical role of type II alveolar epithelial cell homeostasis that provides the basis for the pathogenesis of lung diseases in neonates, infants and, more rarely, in older individuals. Together, advances in understanding the role of the surfactant system in the pathogenesis of acute and chronic lung disease has rendered the term "idiopathic" pulmonary fibrosis or idiopathic interstitial lung disease as obsolete for at least a few of these inherited disorders.

Funding: Supported by: NIH grants 5R01HL38859 and 5R01HL085610

Competing interests: None.

Thorax 2008;63:295-296. doi:10.1136/thx.2007.091231

\section{REFERENCES}

1. Doan ML, Guillerman RP, Dishop MK, et al. Clinical, radiological and pathological features of $A B C A 3$ mutations in children. Thorax 2008;63:366-73.

2. Shulenin S, Nogee LM., Annilo T, et al. ABCA3 gene mutations in newborns with fatal surfactant deficiency. N Engl J Med 2004;350:1296-303.

3. Saugstad OD, Hansen TWR, Rønnestad A, et al. Novel mutations in the gene encoding ATP binding cassette protein member $\mathrm{A} 3(\mathrm{ABCA} 3)$ resulting in fatal neonatal lung disease. Acta Pædiatr 2007:96:185-90. 
4. Garmany TH, Moxley MA, White FV, et al. Surfactant composition and function in patients with ABCA3 mutations. Pediatr Res 2006;59:801-5.

5. Bullard JE, Wert SE, Whitsett JA, et al. ABCA3 mutations associated with pediatric interstitial lung disease. Am J Respir Crit Care Med 2005;172:1026-31.

6. Whitsett JA, Weaver TE. Hydrophobic surfactant proteins in lung function and disease. $N$ Engl J Med 2002;347:2141-8.
7. Nogee LM. Alterations in SP-B and SP-C expression in neonatal lung disease. Annu Rev Physiol 2004;66:601-23

8. Fitzgerald ML, Xavier R, Haley KJ, et al. ABCA3 inactivation in mice causes respiratory failure, loss of pulmonary surfactant, and depletion of lung phosphatidylglycerol. J Lipid Res 2007;48:621-32.

9. Hammel M, Michel G, Hoefer C, et al. Targeted inactivation of the murine $\mathrm{ABCA} 3$ gene leads to respiratory failure in newborns with defective lamellar bodies. Biochem Biophys Res Commun 2007;359:947-51.

10. Cheong $\mathbf{N}$, Zhang $H$, Madesh $M$, et al. ABCA3 is critical for lamellar body biogenesis in vivo. J Biol Chem 2007;282:23811-7.

11. Huqun SI, Miyazawa $\mathrm{H}$, Ishii $\mathrm{K}$, et al. Mutations in the SLC34A2 gene are associated with pulmonary alveolar microlithiasis. Am J Respir Crit Care Med 2007; 175:263-8.

\section{Use of $\beta$ blockers in patients with COPD}

\section{David H Au}

Chronic obstructive pulmonary disease (COPD) is the leading cause of respiratory-related deaths in the USA. ${ }^{1}$ This simple and dramatic statistic, however, does not present the full story. In developed countries, smoking tobacco is the principal cause of COPD and also represents a major risk factor for other conditions such as cardiovascular disease and lung cancer. ${ }^{2}$ One question that remains largely unanswered is how the presence of COPD modifies the treatment of coexisting illnesses such as cardiovascular disease. This question is important because most patients with COPD do not die of COPD but, as demonstrated by randomised trials and observational studies, the principal causes of death are most often listed as cardiovascular-related or lung cancer-related..$^{3-5}$ Tension in treatment decisions often occurs when clinicians must decide about providing patients with treatments that are known to improve outcomes for selected patients while potentially causing harm in others. A paradigm for this dilemma is the use of $\beta$ blockers in patients with COPD.

Beta blockers are first-line therapy for patients with cardiovascular disease and have been shown in randomised clinical trials to reduce mortality. ${ }^{6-8}$ The strongest evidence for the mortality benefit of $\beta$ blockers is in myocardial infarction, ${ }^{6-8}$ ischaemia ${ }^{8}$ and left ventricular systolic dysfunction, ${ }^{910}$ but there are a number of other settings including hypertension, ${ }^{11-13}$ tachyarrhythmia, ${ }^{14}$ perioperative risk modification ${ }^{15}{ }^{16}$ and thyrotoxicosis. ${ }^{17-19}$ Although there are many potential explanations for the reduction in mortality, the benefit is clear. Beta blockers, by their effect

Correspondence to: Dr D H Au, Health Services Research and Development, VA Puget Sound Health Care System, Suite 1400, 1100 Olive Way, Seattle, WA 98101; dau@u.washington.edu on the $\beta$ adrenoceptor, decrease myocardial contractility, chronotropic response and myocardial oxygen demand. ${ }^{20}$ In the setting of myocardial ischaemia, these effects reduce overall myocardial injury. ${ }^{21-23}$ In addition, $\beta$ blockers reduce cardiovascular mortality based on anti-arrhythmic properties and the prevention of malignant arrhythmias. $^{24}{ }^{25}$ Although it may seem reasonable to apply the results of clinical trials to all individuals with the proper indication, the results of clinical trials are generalisable only to those individuals who would have qualified to participate. For most clinical trials of cardiovascular diseases and $\beta$ blockers, participants with obstructive pulmonary diseases were excluded. ${ }^{26}$ This has led to a major gap in our ability to use randomised evidence to support the use of these medications in patients with COPD.

Although last updated some time ago, the most current American College of Chest Physicians guidelines for the treatment of systemic hypertension recommend that $\beta$ blockers are relatively contraindicated in patients with COPD. This opinion is consistent with a more recent review which suggested that these agents should not be used without careful examination of the risk and benefits. ${ }^{27}$ Others, however, suggest that the evidence from short-term randomised studies and observational studies for the safety of at least cardioselective $\beta$ blockers is growing. Meta-analyses of randomised studies suggest that cardioselective $\beta$ blockers are not associated with decrements in symptoms or in forced expiratory volume in $1 \mathrm{~s}\left(\mathrm{FEV}_{1}\right) .{ }^{28}$ Moreover, there are now several observational studies that suggest that $\beta$ blockers may, in fact, reduce mortality among patients with COPD. ${ }^{29-32}$ Given the conflicting recommendations, how do clinicians decide what is the correct course of action? A fair interpretation is that both groups are neither right nor wrong. There is simply a paucity of data to address this question. As a result, clinicians may in fact use $\beta$ blockers in patients with COPD who have appropriate indications, but they do so significantly less often than in patients without COPD.

In this issue of Thorax Dransfield et al ${ }^{33}$ have added to the body of observational studies by demonstrating that patients with COPD who received $\beta$ blockers during a hospital admission had a significant reduction in all-cause mortality (see page 301). Patients were selected to participate in the study if they had a primary diagnosis of COPD or a primary diagnosis of respiratory failure associated with a secondary diagnosis of COPD. The authors identified prescriptions for $\beta$ blockers using the hospital billing records and identified both cardioselective and non-cardioselective agents. Of the 825 patients identified, 142 (17.2\%) had received a $\beta$ blocker and $43(5.2 \%)$ died while in hospital. Patients who had received $\beta$ blockers were older, had more co-morbid illnesses (especially cardiovascular disease), but were similar with regard to markers of severity of pulmonary disease. In an adjusted model, patients who received $\beta$ blockers were nearly $60 \%$ less likely to die in hospital than those who did not receive $\beta$ blockers (adjusted OR 0.39 (95\% CI 0.14 to 0.99$)$ ). This estimate was robust to traditional methods of adjustments including the use of propensity scores. Although spirometric data were available for only one-third of the total cohort, there was no difference in the overall severity of airflow obstruction in those who did and did not receive $\beta$ blockers.

These results are in general agreement with previous studies that have shown a decreased risk of mortality associated with $\beta$ blocker use in patients with COPD. ${ }^{29-32}$ These previous studies have focused on patients with COPD who were discharged after a myocardial infarction and those patients who were being treated for hypertension. The current study by Dransfield et al fits within the range of existing studies but adds the 